\title{
Delayed rhabdomyolysis secondary to anabolic-androgenic steroid use
}

\author{
Authors: Amelia Benjamin, ${ }^{A}$ Alex Anderson ${ }^{B}$ and Sanja Zrelec ${ }^{C}$
}

\begin{abstract}
There are literature reports of anabolic-androgenic steroidinduced rhabdomyolysis. Here we describe a case of a patient presenting with acute confusion and rhabdomyolysis, following an unwitnessed seizure. He had a background of alcohol excess. Three days into his admission he developed dysphagia. At first, the patient denied the use of any medication or drugs. However, he later revealed he had used the anabolic steroid 'Anavar' (oxandrolone) for 60 consecutive days ending a month prior to his admission. The brief seizure was itself unlikely to have resulted in the severity of his rhabdomyolysis. Thus, we report a likely case of delayed rhabdomyolysis secondary to anabolic steroid use. The oropharyngeal dysphagia may be secondary to the rhabdomyolysis; there have been cases of this described in literature.
\end{abstract}

KEYWORDS: Rhabdomyolysis, creatine kinase, anabolic-androgenic steroids, alcohol

DOI: 10.7861/clinmed.2020-0694

\section{Case presentation}

A 35-year-old man was admitted with new-onset confusion and an unwitnessed 'unresponsive' episode while at home. The episode lasted 1 minute and was not associated with tongue-biting or incontinence. He had a history of alcoholism, drinking 3-4 bottles of wine daily. His last drink had been a few days ago, following a week-long 'binge'. He had no other medical conditions and denied the use of any illicit or over-the-counter substances.

Examination was unremarkable other than an unsteady gait and confused speech. He was clinically dry, and tachycardic (heart rate at 106 beats per minute). An initial venous blood gas (VBG) showed a lactate of $10 \mathrm{mmol} / \mathrm{L}$, with a normal $\mathrm{pH}$ and blood glucose of $13 \mathrm{mmol} / \mathrm{L}$. The lactataemia resolved with fluid resuscitation. Bloods revealed a potassium of $2.9 \mathrm{mmol} / \mathrm{L}$, creatine kinase (CK) of 14,469 U/L and alanine aminotransferase (ALT) of $81 \mathrm{U} / \mathrm{L}$. Other liver function tests were within normal range. His renal function was impaired (estimated glomerular filtration rate was $71 \mathrm{~mL} / \mathrm{min} / 1.73 \mathrm{~m}^{2}$; baseline $>90 \mathrm{~mL} / \mathrm{min} / 1.73 \mathrm{~m}^{2}$ ).

Authors: ${ }^{A}$ foundation year-2 doctor, West Middlesex University Hospital, Isleworth, UK; ${ }^{\text {B }}$ foundation year-1 doctor, West Middlesex University Hospital, Isleworth, UK; ${ }^{C}$ consultant, West Middlesex University Hospital, Isleworth, UK
C-reactive protein was $86.4 \mathrm{mg} / \mathrm{L}$. Urine toxicology was positive for benzodiazepines only (although he had received chlordiazepoxide when this was performed).

\section{Diagnosis}

The 'unresponsive episode' was treated as an alcohol withdrawal seizure. A wide differential was proposed for the patient's confusion.

$>$ A post-ictal state.

$>$ Central nervous system (CNS) infection.

$>$ Alcohol withdrawal syndrome.

> Use of an unidentified drug, not identified on the toxicology screen.

The elevated CK confirmed rhabdomyolysis. This was likely to be secondary to either alcohol, drug use or seizure activity.

\section{Treatment}

Initial management was supportive; his potassium was replaced and intravenous (IV) fluids were commenced. He was prescribed Pabrinex, chlordiazepoxide and a Clinical Institute Withdrawal Assessment of Alcohol Scale (CIWA) score was kept. Acyclovir and ceftriaxone were given to cover CNS infection.

\section{Case progression}

A lumbar puncture revealed no evidence of infection. The patient's confusion resolved, and he had no further seizures. His CK initially fell to $8,217 \mathrm{U} / \mathrm{L}$, then rose to $50,800 \mathrm{U} / \mathrm{L}$. This rise was associated with an increment in ALT (from $81 \mathrm{U} / \mathrm{L}$ to $149 \mathrm{U} / \mathrm{L}$ ). Troponin-T was elevated (132 ng/L). The elevated CK, troponin and ALT together with urinalysis (dipstick positive for blood, microscopy negative for red blood cells) were typical of rhabdomyolysis. IV fluids prevented any deterioration in renal function. Table 1 depicts the trend of investigation findings.

On day 3 of admission, the patient began to complain of swallowing difficulties, leg soreness, and difficulty standing from sitting. Examination revealed no gait abnormality or other focal neurology. He was not withdrawing from alcohol clinically; his CIWA score was 0 . Computed tomography of the chest, abdomen and pelvis revealed no masses or pathology. Thyroid function tests and a morning cortisol were within normal range. Liver ultrasound revealed a mild diffuse hepatic steatosis (Fig 1).

By day 4 , his CK had reached 86,350 U/L. On further questioning, the patient confidentially revealed that he had been taking 


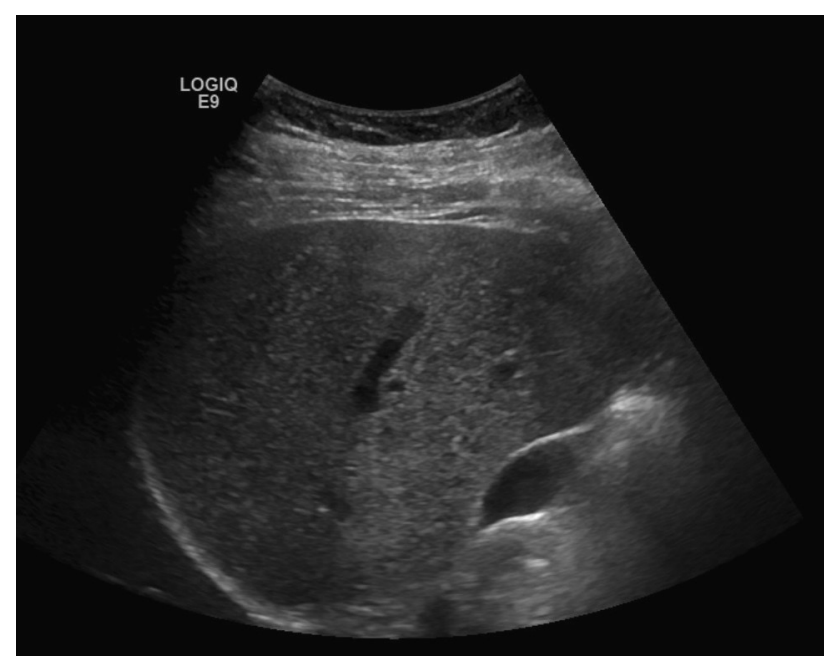

Fig 1. Liver ultrasound revealing a mild diffuse hepatic steatosis.

'Anavar' (oxandrolone), an anabolic-androgenic steroid, for 60 consecutive days, ending his course of the drug a month prior to admission. This had been in an attempt to enhance his gym performance.

IV fluids were continued and, on day 5 of admission, his CK had fallen to 62,700 U/L. His dysphagia had improved. He was discharged on day 6 of admission, when his CK had fallen to 29,400 U/L. He was followed up in our ambulatory emergency care department; his CK had fallen to 2,572 U/L, and his electrolytes were within normal range. Seven days after his initial presentation, he was discharged.

\section{Discussion}

We describe a patient with a background of alcohol abuse who presented with confusion, a progressive development of skeletal muscle fibre rhabdomyolysis and dysphagia. His confusion is likely to be post-ictal.

Rhabdomyolysis is diagnosed when the measured CK reaches five times the upper limit of normal. It is the result of any disease process to cause myocyte lysis. These processes can be split into traumatic or non-traumatic.

His seizure, although a potential cause of traumatic rhabdomyolysis, was not sufficiently prolonged to warrant such an increment in CK. Instead, a non-traumatic cause is more likely: either chronic alcohol excess (there are previous reports of non-traumatic rhabdomyolysis secondary to long-term alcohol abuse) or his use of oxandrolone. ${ }^{1}$

The pathophysiology of alcohol-related nontraumatic rhabdomyolysis is poorly understood. Electrolyte abnormalities, such as hypokalaemia and hypomagnesaemia, may play a role in muscle necrosis in persons with long-term alcoholism. These are likely to be secondary to nutritional deficiency. Our patient was hypokalaemic on admission. This could be the cause of (or a contributing factor to) the development of rhabdomyolysis. Hypokalaemic myopathy itself is a form of toxic myopathy. ${ }^{2}$

A second explanation for the patient's rhabdomyolysis is his heavy use of anabolic-androgenic steroids. There is literature describing rhabdomyolysis and biopsy-proven skeletal muscle necrosis secondary to anabolic steroid use. ${ }^{3}$ The pathophysiology behind anabolic steroid-induced generalised myopathy remains unclear and is mostly demonstrated by animal models. ${ }^{4}$

Anabolic steroids have a myotrophic effect and are used to increase muscle mass in patients with major burns, or chronic wasting conditions. ${ }^{5,6}$ Although useful in medicine, they have several unwanted side effects, including rhabdomyolysis, hepatic steatosis (confirmed on liver ultrasound in this patient) and cardiomyopathy. If anabolic steroid use is the cause of our patient's rhabdomyolysis, this would be the first report of delayed rhabdomyolysis secondary to anabolic steroids (the patient had stopped using steroids 1 month previously).

The patient's dysphagia remained unexplained after clinical imaging. Its onset coincided with myalgia and increasing CK, suggesting the dysphagia may be secondary to rhabdomyolysis; there have been cases of this described before. ${ }^{7}$ This is the first case of dysphagia secondary to anabolic steroid use.

This case prompts further investigation into the musculoskeletal side effects of anabolic-androgenic steroids. It highlights the benefits of taking a comprehensive history.

\section{Key points}

> Anabolic-androgenic steroids are associated with the delayed onset of skeletal muscle breakdown / rhabdomyolysis.

> This is one of the few cases to describe dysphagia associated with rhabdomyolysis.

> Aggressive fluid resuscitation prevented renal impairment in this patient and is important in the treatment of rhabdomyolysis.

\section{Supplementary material}

Additional supplementary material may be found in the online version of this article at www.rcpjournals.org/clinmedicine: S1 - Trend of investigation findings throughout the patient's admission.

\section{References}

1 Qiu LL, Nalin P, Huffman Q et al. Nontraumatic rhabdomyolysis with long-term alcohol intoxication. J Am Board Fam Pract 2004; 17:54-8.

2 Pasnoor M, Barohn RJ, Dimachkie MM. Toxic myopathies. Neurol Clin 2014;32:647-70, viii.

3 Hughes M, Ahmed S. Anabolic androgenic steroid induced necrotising myopathy. Rheumatol Int 2011;31:915-7.

4 Abu-Shakra S, Alhalabi MS, Nachtman FC et al. Anabolic steroids induce injury and apoptosis of differentiated skeletal muscle. J Neurosci Res 1997:47:186-97.

$5 \mathrm{Li} \mathrm{H}, \mathrm{Guo}$ Y, Yang Z et al. The efficacy and safety of oxandrolone treatment for patients with severe burns: A systematic review and meta-analysis. Burns 2016;42:717-27.

6 Orr R, Fiatarone Singh M. The anabolic androgenic steroid oxandrolone in the treatment of wasting and catabolic disorders: review of efficacy and safety. Drugs 2004;64:725-50.

7 Norsworthy ], Kurtis H. Dysphagia in rhabdomyolysis: 2643. Am J Gastroenterol 2016;111:S1332.

Address for correspondence: Dr Amelia Benjamin, West Middlesex University Hospital, Twickenham Road, Isleworth, Middlesex TW7 6AF, UK.

Email:ameliabenjamin@live.co.uk 